\title{
Le calvaire du sujet « traduit »
}

Version française

Lawrence Phillips

\section{(2) OpenEdition \\ Journals}

Electronic version

URL: http://journals.openedition.org/transtexts/166

DOI: $10.4000 /$ transtexts. 166

ISSN: 2105-2549

Publisher

Gregory B. Lee

\section{Printed version}

Date of publication: 1 May 2006

Number of pages: $26-28$

ISSN: 1771-2084

\section{Electronic reference}

Lawrence Phillips, «Le calvaire du sujet « traduit » , Transtext(e)s Transcultures 跨文本跨文化 [Online], 1 | 2006, Online since 13 September 2009, connection on 21 April 2019. URL : http:// journals.openedition.org/transtexts/166; DOI : 10.4000/transtexts.166 


\section{The agony \\ of the translated subject \\ by Lawrence Phillips}

Preparing to write this response to the first edition of Transtext(e)s Transcultures little over a month after the London bombings, I am unable to dismiss from my mind how the political debate in Britain has quickly turned to the formerly unquestioned principle of multiculturalism in the United Kingdom. The debate has not always been measured and in all too many cases, the xenophobia and suppressed racism that underlies many sectors of British society has re-emerged, permeating the void opened by the political and social crisis in the wake of the atrocities. What measured reaction there has been has often from Britain's ethnic minorities, particularly British Muslims of varying origins, despite a wave of racist attacks and abuse in the wake of the bombings. Yet the rhetoric from politicians and the press does not match the mood of the majority of the population. Indeed, a survey conducted for the BBC by MORI only a month after the 7 th July bombings demonstrated that nationally, $62 \%$ of the population felt that the multicultural identity of the UK enhanced British society, which rose to a startling $87 \%$ among British Muslims. ${ }^{1}$ Positively this survey suggests that multiculturalism is an entrenched fact of British life, even when conducted in conditions unpropitious to a liberal response. Yet what does multicultural mean in contemporary society ? Some $58 \%$ of the respondents to the same BBC/MORI survey indicated that people who came to live in Britain should adopt the values and traditions of British society.

Clearly, at least for a significant number of the respondents, "multicultural" has become confused with "multiethnic". Culture, in a manner any Victorian essentialist would recognise, has collapsed into race. Such contradictions radically undermine the political ideal of many cultures and traditions living in peaceful coexistence owing allegiance to the same national and political institutions. At times of crisis, the underlying strains are ever likely to emerge, even in a country with a ready-made trans-national, if not trans-cultural, "national" identity available in Britishness. This very issue goes to the heart of this new journal's focus on diaspora, transnational identities, hybridity, and cultural translation. The identification of these specific phenomena rather than collecting them under some broader category is, I feel, of critical significance and is related to more practical issues of national and international cultural translation and understanding that the $\mathrm{BBC} / \mathrm{MORI}$ survey raises over 'multiculturalism' and ethnicity where academics should be providing a lead. It also points to increasing challenges faced by more totalising terminology such as post-colonialism. This is not to dismiss any of the important work associated with that banner in the least, but I do wish to point to the problems with the two principal historio-critical narratives the term establishes.

The first narrative maps out the first flush of optimism, both political and cultural, in response to independence from the vast nineteenth-century empires that largely collapsed after the

\footnotetext{
1 http://news.bbc.co.uk
} 
Second World War. Optimism not misplaced at the time, but surely brought increasingly into question by the events of recent years. The political, economic, and social reality is that those empires were only in retreat on one front, leaving behind a world system predetermined to favour the former imperialists. The "post" in post-colonialism has in practice proved to be contingent at best, and at worst entirely idealistic. Indeed, the turn of the twentieth century has seen the re-emergence of direct imperial action in Iraq, Afghanistan and dreadful ethnic repression in the Sudan, Indonesia and elsewhere, and a new revisionist history of colonialism promoted by publications like Nail Ferguson's popular history Empire: How Britain Made the Modern World (2003), which spawned a successful TV series in the UK. Even more recently, calls by right-wing politicians in the UK for positive teaching of Britain's imperial history in schools has been on the political agenda. On other fronts, objections to Turkish entry to the European Union seem increasing racially based, while many of the disparate groups that protest against the social, economic, and cultural affects of globalism seem to blissfully unaware that they are, in fact, continuing a long (and arguably less historically conscious) struggle against colonialism.

The second narrative establishes a political idealism that coalesces around and celebrates ideas like the "in-between", "hybridity", and, indeed, "multiculturalism". These concepts have been taken to be indicative of creative cultural syntheses. What such optimism lacks is an acknowledgment of the pain, resistance, confusion and disillusionment that such experiences can create in practice. The process of cultural syntheses is a struggle at best that can lead to a dubious accommodation with the local hegemonic culture. What is the lived experience of "hybridity" or being culturally, socially and politically located "in-between"? Stuart Hall, writing just a decade ago in 1995, is not untypical of the political and social idealism associated with this narrative:

They are people belong to more than one world, speak more than one language (literally and metaphorically); inhabit more than one identity, have more than one home; who have learned to negotiate and translate between cultures and who, because of this are irrevocably the product of several interlocking histories and cultures have learned to live with, and indeed to speak from difference. ?

The British Muslim bombers of 7th July are just such translated individuals but, one presumes, felt strongly enough about the plight of their co-religionists around the globe to wish to kill themselves and others in protest. That doesn't legitimise their act, and one might, as the British Prime Minister has, attempt to dismiss them as the misguided acts of a group of young men seduced by a pernicious ideology peddled by fanatics. Yet according to the immigrant parents of the alleged bombers, rather than dangerous deviants several of them were striking only by their apparent accommodation with life in Britain. On reflection though, and subject to any other explanation as to the motivations of these otherwise well-adjusted individuals, some pause for thought must ensue that reflects not only on act and reaction, commentary and theory, but also on the lived experience of cultural translation, hybridity, and the "in-between" which clearly left a cultural void for these young men. Clearly the narratives available under "post-colonialism", of independence, cultural reclamation, and the "joy" of the hybridity, or even multiculturalism are not adequately engaged with the experiences of these second generation British Muslims.

2 Stuart Hall, "New Cultures for Old" in D. Massey and P. Jess (eds.), A Place in the World: Places, Culture and Globalisation, Oxford, Oxford University Press, 1995, p. 47. 
A new positive and intellectually engaged discourse is desperately needed to counter the increasingly reactionary politics of our times. To continue to subsume hybrid, transnational, and transcultural identities under a rubric that fails to offer an insight into the lived experiences of those for whom daily life is a constant negotiation and re-negotiation would point to the risk of serious academic neglect. The specific issues of formation, struggle, politics, representation, and expression of working accommodations between cultures needs to be urgently reappraised. Such a project must be multilingual, multicultural, transnational, and interdisciplinary. I am encouraged by the timely publication of Transtext(e)s Transcultures and feel confident that the forum it provides will foster such exchanges. Such exchanges are ever urgently needed in an era of increasing uncertainty, violence, misunderstanding, and ill-informed opinion in which culture can be confused with racial difference in an apparently liberal response to a terrible atrocity without any sense of the social and political regression that this in fact represents.

\section{Le calvaire du sujet « traduit »}

Environ un mois après les attentats de Londres et alors que je me prépare à rédiger cette réponse au premier numéro de Transtext(e)s Transcultures, je ne peux m'empêcher de penser à la vitesse à laquelle le débat politique en Grande-Bretagne s'en est pris au principe jusqu'alors inattaquable du multiculturalisme au Royaume-Uni. Ce débat n'a pas toujours été serein et trop souvent la xénophobie et le racisme latents dans bien des secteurs de la société britannique ont refait surface, s'insinuant dans le vide créé par la crise sociale et politique engendrée par les atrocités. S'il y a eu une réaction mesurée, elle est venue des minorités ethniques de Grande-Bretagne, en particulier des musulmans britanniques d'origines diverses, en dépit d'une série d'agressions racistes faisant suite aux attentats. Pourtant la rhétorique déployée par la presse et les politiciens ne correspond pas aux sentiments de la majorité. Ainsi, un sondage mené par MORI pour la BBC un mois seulement après les attentats du 7 juillet, indique que $62 \%$ des gens estimaient que l'identité multiculturelle du Royaume-Uni était un atout pour la société britannique, allant jusqu'à $87 \%$ des opinions des musulmans britanniques. ${ }^{3} \mathrm{Ce}$ sondage démontre de manière positive combien le multiculturalisme est ancré dans la vie britannique, ce, même dans les conditions les moins favorables à une réponse libérale. Mais que signifie multiculturel dans la société contemporaine ? Environ $58 \%$ des personnes interrogées dans le même sondage $\mathrm{BBC} / \mathrm{MORI}$ ont déclaré que les personnes qui venaient vivre en Grande Bretagne devaient adopter les traditions et les valeurs de la société britannique.

II est clair qu'une grande partie des personnes interrogées confond «multiculturel» et "multiethnique ». La culture a été ramenée à la race, d'une manière qu'aurait applaudi 
n'importe quel essentialiste victorien. De telles contradictions sapent sérieusement l'idéal politique de nombreuses cultures et traditions qui coexistent pacifiquement en respectant les mêmes institutions nationales et politiques. En temps de crise, les tensions sous-jacentes risquent toujours de remonter à la surface, même dans un pays comme la Grande-Bretagne où l'identité nationale est naturellement transnationale, sinon transculturelle. Cette question se trouve au centre des préoccupations de diaspora, d'identités transnationales, d'hybridité et de traduction culturelle de ce journal . Identifier ces phénomènes spécifiques plutôt que de les rassembler à l'intérieur d'une catégorie plus vaste me semble d'une importance critique et touchant à des problèmes plus pratiques de traduction et de compréhension culturelle nationale et internationale que ceux soulevés par le sondage BBC/MORI sur le multiculturalisme et l'ethnicité domaines dans lesquels les universitaires devraient montrer la voie. De plus, cette identification souligne les défis toujours plus nombreux posés aux terminologies plus totalisantes telles que celle de post-colonialisme. II ne s'agit en aucun cas de nier l'importance du travail associé à ce titre, mais d'évoquer les problèmes liés aux deux principaux narratifs historico-critiques établis par ce terme.

Le premier d'entre eux trace les grandes lignes de l'élan d'optimisme, à la fois politique et culturel, qui a fait suite à l'indépendance des vastes empires du XIXe siècle qui s'effondrèrent pour la plupart après la deuxième guerre mondiale. Cet optimisme qui, à l'époque n'était certes pas déplacé, s'est vu de plus en plus remis en question par les événements de ces dernières années. La réalité politique, sociale et économique est telle que la retraite de ces empires ne fut que momentanée, qu'ils laissèrent en place dans leur sillage un système mondial prédisposé en faveur des anciens impérialistes. Dans la pratique le "post» de postcolonialisme s'est avéré au mieux contingent et au pire entièrement idéaliste. Ainsi, la fin du XXe siècle a-t-elle vu le retour de l'action impériale directe en Iraq, en Afghanistan, d'effroyables répressions ethniques au Soudan, en Indonésie et ailleurs, ainsi que l'émergence d'une nouvelle histoire révisionniste du colonialisme promue par des ouvrages comme I'histoire populaire de l'Empire de Nail Ferguson : How Britain Made the Modern World (2003) qui a donné lieu à une adaptation télévisée très applaudie au Royaume-Uni. Encore plus récemment, des politiciens de droite au Royaume-Uni ont demandé que l'enseignement de I'histoire impériale britannique soit enseignée sous un angle positif dans les écoles. Par ailleurs, les objections à l'entrée de la Turquie dans l'Union Européenne se font de plus en plus racistes, alors que bien des groupes disparates qui s'élèvent contre les impacts sociaux, économiques et culturels de la mondialisation semblent ignorer complètement qu'ils sont en réalité dans la lignée d'un long (et sans doute moins conscient historiquement) combat contre le colonialisme.

Le deuxième de ces narratifs établit un idéalisme politique qui célèbre et se fond dans des idées comme "l'entre-deux ", "l'hybridité » et bien entendu "le multiculturalisme ». On a considéré ces concepts comme révélateurs de synthèses culturelles créatives. Ce qui manque à un tel optimisme c'est la reconnaissance de la souffrance, de la résistance, du trouble et du désenchantement qu'occasionnent en pratique de telles expériences. Le processus de synthèse culturelle est au mieux un combat qui peut aboutir à des compromis douteux avec la culture hégémonique locale. Quelle est l'expérience réelle de l'« hybridité » ou celle d'être « entre-deux» aux points de vue culturel, social et politique ? Ce qu'écrivait Stuart Hall, il y a juste dix ans, en 1995, n'est pas sans rappeler l'idéalisme politique et social inhérent à ce narratif : 
॥ y a des gens qui appartiennent à plus d'un monde, qui parlent plus d'une langue (littéralement et métaphoriquement) ; qui habitent plus d'une identité, qui possèdent plus d'une maison ;qui ont appris à négocier et à passer d'une culture à l'autre et qui, en raison de cela sont irrévocablement le produit de plusieurs histoires et cultures imbriquées les unes dans les autres et qui ont appris à vivre avec et, qui plus est, à parler, du point de vue de la différence. 4

Les poseurs de bombe musulmans britanniques du 7 juillet sont ainsi de ces sujets traduits mais qui, semblerait-il, se sont sentis assez touchés par la misère de leurs co-religionnaires dans le reste du monde pour désirer se suicider en guise de protestation. Cela ne justifie en rien leur acte, et l'on pourrait, comme l'a fait le Premier Ministre britannique, n'y voir que les actes malavisés d'un groupe de jeunes hommes séduits par une idéologie pernicieuse colportée par des fanatiques. Pourtant, selon les parents des prétendus poseurs de bombes, ceux-ci ne se distinguaient que par leur apparente intégration à la vie en Grande-Bretagne. II s'agit toutefois, et en tenant compte de toute autre explication quant aux motivations de ces individus par ailleurs bien insérés dans la société, de réfléchir non seulement aux actes et à la réaction, au commentaire et à la théorie qui s'ensuivirent, mais aussi à l'expérience vécue de la translation culturelle, de l'hybridité, et de «l'entre-deux » qui ont de toute évidence donné lieu pour ces jeunes hommes à un vide culturel. II est clair que les narratifs postcoloniaux d'indépendance, de reconquête culturelle et des «joies » de l'hybridité sont très loin de l'expérience de cette seconde génération de musulmans britanniques.

Un nouveau discours positif engagé politiquement et intellectuellement tente désespérément de résister aux politiques toujours plus réactionnaires de notre temps. Si l'on persiste à subsumer les identités hybrides, transnationales et transculturelles sous une rubrique qui ne donne aucune idée des expériences vécues de ceux dont la vie quotidienne n'est que négociation et renégociation, on est en passe de commettre une grave négligence intellectuelle. II est urgent de réévaluer les problèmes spécifiques de formation, de lutte, de politique, de représentation ainsi que l'expression de compromis satisfaisants entre les cultures. Un tel projet doit être multilingue, multiculturel, transnational et interdisciplinaire. C'est pourquoi je suis encouragé par la publication opportune de Transtext(e)s Tanscultures et que je suis convaincu que le forum qu'elle crée promouvra de tels échanges. Echanges requis d'urgence à une époque d'incertitude croissante, de violence, d'incompréhension et d'opinion mal informée, dans laquelle on confond culture et différence raciale en réponse, d'apparence libérale, à une terrible atrocité sans aucune conscience de la régression sociale et politique qu'elle représente effectivement.

\section{被译主 体的焦虑}

伦敦爆炸事件发生后, 英国政治辩论论题很快转向了以前从未受质疑的多元 文化主义。我动手为《跨文本 跨文化》第一期杂志写这篇文章正是在事件发 
生后不到一个月, 因此我根本无法将思绪从这些辩论中抽离。辩论并不总是有 分寸的，而且随着流血事件发生之后的政治和社会危机，英国社会当中潜在 的“恐外症”和克制种族主义再次抬头。虽然爆炸事件引发了一系列的种族袭击 和报复，但是英国的少数族裔（尤其是来自不同国家的英国穆斯林）的反应却 显得非常平静。但是政客们和媒体的话语和大部分人的心情并不吻合。BBC和 MORI 在七月七日爆炸事件发生后一个月的一份访问调查显示，全国 $62 \%$ 的人 认为英国的多元文化身份对社会有利; 而英国持相同意见的穆斯林人数高达 $87 \%$ 。5 虽然这份调查展开的时间并不是最适当的，这个时候并不利于人们畅所 欲言，但是调查结果指明多元文化性是英国社会生活的组成部分之一。那么多 元文化对于现代社会而言有什么含义? $58 \%$ 的受访者认为来到英国居住的人应 该接受并适应英国社会的传统和价值观。

显而易见，受访者当中至少有相当数量的人已经把“多元文化”和“多种族”多 元族群混淆。文化, 以任何维多利亚（Victorian）本质主义者都可以辩认的方 式, 沦为种族问题。这种混淆严重地破坏了这么一种政治理想: 多种文化和传 统和平共处, 并忠于相同的国家和政治机构。危机出现时, 潜藏的问题非常 可能会涌现，虽然英国的国家身份“英国性”早已经是跨国的，即使不是跨文化 的。这个问题正是本杂志的焦点所在：离散、跨国身份、混杂性和文化翻译。 对这些具体现象进行具体区分，而不是用一个宽泛的类别硬套，我认为其意义 非同一般，并与BBC/MORI有关“多元文化主义”和“族群性”的调查而引发的 一些更具体的问题，例如国家和国际文化翻译以及理解等有关，而我认为学术 界应该在这些问题上起到引领作用。一些覆盖性术语，例如后殖民主义，面对 着越来越多的挑战，这种具体区分也指向了这些挑战。但这并不意味着否定与 这一术语相关的重要工作, 我只是想点明这一术语所建立的两个主要的历史一 批判性 (historio-critical) 叙述的问题。

第一种叙述主要勾画了第二次世界大战以后，随着十九世纪庞大帝国的瓦解， 为响应脱离于帝国的独立而涌现的第一股乐观主义情绪: 政治和文化的乐观主 义。这种乐观主义并非不合时宜，但是近年来的许多事情使人们对乐观主义 不断地进行反思。政治、经济和社会现实表明，这些帝国撤退后留下的世界 体系，早已经被预设好，这个世界依然支持帝国主义者。“后殖民”中的“后”最 好的情况不过是一种偶然的的实践, 而最坏的情况则是仅为一种理想主义。二 十一世纪初，我们见证了在伊拉克和阿富汗发生的直接的帝国主义行径，在苏 丹、印尼和其它地方发生的族群压迫，还有一些出版物鼓吹以修正主义观点 看待殖民历史，就如傅格逊（Nail Ferguson）在其畅销书《英国如何建造现代世 界》（2003）中所倡导的一样，他的书已在英国改编成电视连续剧，大受百姓 喜爱。最近, 英国右翼政客呼吁英国学校在传授知识过程中应该正视英国的帝 国历史，这已经被提上政治日程。在其它方面，反对土耳其加入欧盟的意见似 乎越来越明显地呈现出种族主义倾向。许多独立组织抗议全球主义对社会、经 济和文化带来的影响，但是他们丝毫没有意识到，他们实际上是在推动一场反 殖民主义的持久战。

第二种叙述建立了一种政治理想主义, 它是一些思想的融合, 而且为之而欢 呼，例如“夹缝性”（inbetween）、“混杂性”（hybridity）和“多元文化主义”等。这 些概念一直被认为具有创造性文化综合体的意味。这种乐观主义缺乏的是对 这些经验在实践过程中造就的痛苦、抵抗、混淆和幻灭的认识。文化综合体的 形成其实是一个抗争的过程，但其结果是其它文化不得不迁就迎合本土霸权文 化, 但是这种迎合和迁就值得思考和怀疑。“混杂性”或者说在文化上、社会上

5 http://news.bbc.co.uk; MORI: “市场与舆论调查国际”(Market \& Opinion Research International)

一家国际性的市场与舆情调查公司——译者注。 
和政治上被置于二者的夹缝里是怎样一种经历呢? 仅在十年前, 即1995年, 斯 图亚特. 霍尔 (Stuart Hall)写过的下述文字, 正是与这种叙述相关的政治和社会理 想主义的最佳案例。

他们这些人属于多个世界，使用多种语言（从字面上和从比喻意义上来理 解），他们拥有多重身份，拥有多个家庭。他们已经学会了如何在文化之间进 行沟通和传译, 因此他们是同种相互连接的多重历史和文化的产物, 而他们已 经学会如何容忍差异, 以及如何从差异的角度来说话。6

七月七日引发爆炸的英国穆斯林正是这一类被译主体。人们认为, 这些穆斯林 对世界各地拥有与之相同信仰的人深怀同情，因此他们用杀害他人和自己的方 式来发出抗议。这种说法并不能成为他们行为的托辞。人们也许会像英国首相 一样, 认为他们这群年青人的错误行为, 是受了宗教狂热者所兒售的意识形态 之毒所致。然而根据涉嫌犯父母所言，其中的一些人并不是危害社会的离经叛 道者, 他们的动机, 是为了表明他们与英国社会只属于表面的融合。在思考这 些年青人的动机之时, 我们不仅仅要考虑行为和反应、评论和理论, 我们还必 须考虑文化翻译、混杂性以及介于夹缝间等经历，因为这些经历让这些年青人 产生空虚感。在“后殖民主义”之下的叙述、独立、文化垦荒、混杂性的欢乐甚 至是多元文化主义，并不足以说明这些第二代英国穆斯林的体验。

一种新的、积极的, 并在知识分子界被广泛应用的话语, 绝对需要考虑我们这 个时代日益增长的反动政治。继续用单一的标签来覆盖混杂的、跨国的和跨文 化的众多主体, 将会导致严重的学术疏忽的危险。这些主体的最实在的体验就 是，他们的日常生活是一场持久的协商、再协商的过程。而单一的标签并不能 为这一体验提供任何洞见。形成、挣扎、政治、再现和文化间的迁就等等详细 的问题急需被重新评估。这项重估工程必须是多语的、多文化的、跨国的和跨 学科的。《跨文本跨文化》及时出版让我深受鼓舞。我非常有信心, 该杂志 的论坛将会推动交流。在这个年代, 不安、暴力、误解和错误意见越来越多。 在这个年代, 人们在回应一件可怕的暴行的时候, 将文化与种族差异混为一 谈，而丝毫没有意识到社会和政治的倒退。在这么一个年代，该杂志所推动的 交流显得尤为迫切。 